\title{
SHADOWING ORBITS OF ORDINARY DIFFERENTIAL EQUATIONS ON INVARIANT SUBMANIFOLDS
}

\author{
BRIAN A. COOMES
}

\begin{abstract}
A finite time shadowing theorem for autonomous ordinary differential equations is presented. Under consideration is the case were there exists a twice continuously differentiable function $g$ mapping phase space into $\mathbb{R}^{m}$ with the property that for a particular regular value $\boldsymbol{c}$ of $g$ the submanifold $g^{-1}(\boldsymbol{c})$ is invariant under the flow. The main theorem gives a condition which implies that an approximate solution lying close to $g^{-1}(\boldsymbol{c})$ is uniformly close to a true solution lying in $g^{-1}(\boldsymbol{c})$. Applications of this theorem to computer generated approximate orbits are discussed.
\end{abstract}

\section{INTRODUCTION}

Let $f$ be a $C^{2}$ vector field on $\mathbb{R}^{n}$ and let $g: \mathbb{R}^{n} \rightarrow \mathbb{R}^{m}$ be class $C^{2}$. Let $\boldsymbol{c}$ in $\mathbb{R}^{m}$ be a regular value of $g$ with

$$
\Lambda=\left\{\boldsymbol{y} \in \mathbb{R}^{n}: g(\boldsymbol{y})=\boldsymbol{c}\right\} .
$$

Suppose that for all $\boldsymbol{y}$ in $\Lambda$ we have

$$
D g(\boldsymbol{y}) f(\boldsymbol{y})=\mathbf{0} .
$$

Thus $\Lambda$ is a smooth $n-m$-dimensional submanifold of $\mathbb{R}^{n}$ and invariant under the flow $\varphi$ of the autonomous system

$$
\dot{\boldsymbol{y}}=f(\boldsymbol{y}) .
$$

In this note we develop a theorem which can be used to prove that a $\delta$-pseudo orbit, or approximate solution, for (1.1) which is close to $\Lambda$ is shadowed by, or is uniformly close to, an orbit of (1.1) which lies in $\Lambda$. Furthermore, the hypotheses of the theorem are presented with an eye toward computer verification. The theorem is similar to the finite time shadowing theorems of Coomes, Koçak, and Palmer [7], [10], but takes into account information provided by $g$ and allows one to say more about the shadowing orbit, namely that it lies in $\Lambda$.

Vector fields possessing first integrals, such as Hamiltonian vector fields, are examples of vector fields for which one might find suitable functions $g$, but in our case we require only that a particular level set of $g$ be invariant under the flow. For example, Poincaré [18] studied the behavior at infinity of flows for polynomial vector fields on $\mathbb{R}^{2}$ by means of central projection onto the sphere $S^{2}$ tangent to the plane at the origin. Under this projection the equator in $S^{2}$ corresponds to infinity

Received by the editors May 17, 1995.

1991 Mathematics Subject Classification. Primary 34A50; Secondary 65L70.

Key words and phrases. Ordinary differential equations, shadowing, Hamiltonian systems, first integrals, invariant manifolds.

(C)1997 American Mathematical Society 
in $\mathbb{R}^{2}$. With $S^{2}$ identified with the unit sphere in $\mathbb{R}^{3}$, a natural scaling arises under which the projected vector field on $S^{2}$ extends to a polynomial vector field on $\mathbb{R}^{3}$. The sphere $S^{2}$ is an invariant set for this new vector field, but $g(\boldsymbol{x})=x_{1}^{2}+x_{2}^{2}+x_{3}^{2}$ need not be a first integral. See also González Velasco [14] for an extension of this projection technique to higher dimensions and a corresponding study of behavior at infinity.

As pointed out by Dawson et al. [12], one obstruction to shadowing occurs when a finite time Lyapunov exponent fluctuates about zero. Certainly one would expect to encounter this situation when phase space is the union of a family of compact level sets of a first integral and the level set of interest contains no fixed point. The shadowing method proposed in this paper compensates for this in the way it takes into account the information provided by such a first integral.

The paper is organized as follows. In section 2 we give a brief history of related shadowing results. Then in section 3 we present our basic definitions and the main theorem. Our main impetus for presenting our result is for the purpose of analyzing specific computer generated numerical solutions of ordinary differential equations. Hence in section 4 we discuss the application of the main result to such computer generated solutions. Finally, in section 5 we give a proof of the main theorem. The issue of roundoff is not addressed in this note but will be addressed in a subsequent publication where the methods described here will be applied to the Maxwell-Bloch [1], [17] system - a five dimensional system which possesses two first integrals.

\section{BACKGROUND FOR SHADOWING}

Many of the systems of ordinary differential equations that are interesting from dynamical or practical points of view are intractable from a computational point of view. That is, for many systems, such as chaotic systems, it is quite difficult to numerically compute an approximate solution of an initial value problem which is close to the true solution over a long time interval. This task becomes generally impossible if one fixes the precision of the numerical representation of real numbers and allows the time interval to become arbitrarily long.

This still leaves open the question: Do numerically generated approximate solutions of chaotic systems reflect true dynamic characteristics? Or, more specifically, given an approximate solution, does there exist some true solution that is uniformly close to the approximate solution? Insight into the answers to these questions came in the Shadowing Lemma of Anosov [2] and Bowen [3] which applies to hyperbolic sets of diffeomorphisms and in this case answered the questions in the affirmative over infinitely long intervals. Franke and Selgrade [13] and later Coomes, Koçak, and Palmer [9] extended this result to hyperbolic sets of vector fields.

That a particular set is a hyperbolic set for a dynamical system is, at best, difficult to verify. However, Hammel, Yorke, and Grebogi [15], [16], Sauer and Yorke [19], Chow and Palmer [4], [5], Chow and Van Vleck [6], and Coomes, Koçak, and Palmer [11] have all investigated finite time shadowing for discrete dynamical systems where one relaxes the hyperbolicity condition and considers solutions over long intervals. Coomes, Koçak, and Palmer [7], [10] studied finite time shadowing for vector fields. An offshoot of their work is their technique [8] for giving a computer generated proof of the existence of a periodic solution of an ordinary differential equation. 


\section{The MAin Theorem}

In this section we give formal definitions, suitable in the context of ordinary differential equations, of a pseudo orbit and of shadowing. We next catalog the various quantities one needs to calculate and examine in order to apply our main result. Then this section concludes with our main result, Theorem 3.1. Throughout this paper, unless otherwise stated, we use the Euclidean norm for vectors and the relevant operator norm for matrices and linear operators. Without loss of generality $\boldsymbol{c}=\mathbf{0}$ and we take division by zero to yield $+\infty$. We adopt the following conventions in our notation: quantities which are presumed to be large, but not too large, are in upper case gothic; quantities which are presumed to be small, but not too small, are in lower case gothic; quantities that are presumed to be quite small are expressed as a variation of $\delta$; quantities that are related to the closeness of our approximate orbit to a true orbit are expressed as a variation of $\varepsilon$. We also identify linear operators between finite dimensional vector space with their matrix with respect to the canonical coordinates.

Definition. For a given positive number $\delta$, a sequence of points $\left\{\boldsymbol{y}_{k}\right\}_{k=0}^{N}$ is said to be a $\delta$-pseudo-orbit of (1.1) if there is an associated sequence $\left\{h_{k}\right\}_{k=0}^{N-1}$ of positive times such that

$$
\left\|\boldsymbol{y}_{k+1}-\varphi^{h_{k}}\left(\boldsymbol{y}_{k}\right)\right\| \leq \delta \quad \text { for } k=0, \ldots, N-1 .
$$

The following is our formal definition of shadowing.

Definition. For a given positive number $\varepsilon$, an orbit of (1.1) is said to $\varepsilon$-shadow a $\delta$-pseudo-orbit $\left\{\boldsymbol{y}_{k}\right\}_{k=0}^{N}$ with associated times $\left\{h_{k}\right\}_{k=0}^{N-1}$ if there are points $\left\{\boldsymbol{x}_{k}\right\}_{k=0}^{N}$ on the true orbit and times $\left\{t_{k}\right\}_{k=0}^{N-1}$ with $\varphi^{t_{k}}\left(\boldsymbol{x}_{k}\right)=\boldsymbol{x}_{k+1}$ such that

$$
\left\|\boldsymbol{x}_{k}-\boldsymbol{y}_{k}\right\| \leq \varepsilon \text { and }\left|t_{k}-h_{k}\right| \leq \varepsilon
$$

The quantity $\varepsilon$ is said to be the shadowing distance and the quantity $\sum_{k=0}^{N-1} t_{k}$ is said to be the shadowing time.

Suppose that $\left\{\boldsymbol{y}_{k}\right\}_{k=0}^{N}$ is a $\delta$-pseudo orbit for (1.1) with associated times $\left\{h_{k}\right\}_{k=0}^{N-1}$. Furthermore, suppose that for $k=0, \ldots, N$ the function $g$, whose zero set is the invariant set $\Lambda$, satisfies

$$
\left\|g\left(\boldsymbol{y}_{k}\right)\right\| \leq \delta \quad \text { and } \quad \operatorname{rank}\left(f\left(\boldsymbol{y}_{k}\right) \mid D g\left(\boldsymbol{y}_{k}\right)^{*}\right)=m+1
$$

where ${ }^{*}$ denotes transpose. Let $\left\{Y_{k}\right\}_{k=0}^{N-1}$ be a sequence of $n \times n$ matrices such that

$$
\left\|Y_{k}-D \varphi^{h_{k}}\left(\boldsymbol{y}_{k}\right)\right\| \leq \delta \quad \text { for } k=0, \ldots, N-1 .
$$

Next let $r=n-m-1$. Choose an $n \times r$ matrix $\widehat{S}$ such that the matrix

$$
\left(f\left(\boldsymbol{y}_{0}\right)\left|D g\left(\boldsymbol{y}_{0}\right)^{*}\right| \widehat{S}\right)
$$

is invertible, let $Q_{0} R_{0}$ be a $\mathrm{QR}$ decomposition of (3.4), and let

$$
Q_{0}=\left(f\left(\boldsymbol{y}_{0}\right) /\left\|f\left(\boldsymbol{y}_{0}\right)\right\|\left|T_{0}\right| S_{0}\right)
$$

where $T_{0}$ and $S_{0}$ are $n \times m$ and $n \times r$ respectively. Then choose subsequent $n \times m$ matrices $T_{k+1}$, and $n \times r$ matrices $S_{k+1}$ for $k=0, \ldots N-1$ by computing

$$
\begin{aligned}
\left(f\left(\boldsymbol{y}_{k+1}\right)\left|D g\left(\boldsymbol{y}_{k+1}\right)^{*}\right| Y_{k} S_{k}\right) & =Q_{k+1} R_{k+1} \\
= & \left(f\left(\boldsymbol{y}_{k+1}\right) /\left\|f\left(\boldsymbol{y}_{k+1}\right)\right\|\left|T_{k+1}\right| S_{k+1}\right) R_{k+1}
\end{aligned}
$$

where $Q_{k+1} R_{k+1}$ is a $\mathrm{QR}$ decomposition. 
Let

$$
A_{k}=S_{k+1}^{*} Y_{k} S_{k} \quad \text { for } k=0, \ldots, N-1
$$

and define a linear operator $L:\left(\mathbb{R}^{r}\right)^{N+1} \rightarrow\left(\mathbb{R}^{r}\right)^{N}$ by, given $\boldsymbol{\xi}=\left\{\boldsymbol{\xi}_{k}\right\}_{k=0}^{N}$ in $\left(\mathbb{R}^{r}\right)^{N+1}$,

$$
[L \boldsymbol{\xi}]_{k}=\boldsymbol{\xi}_{k+1}-A_{k} \boldsymbol{\xi}_{k} \quad \text { for } k=0, \ldots, N-1 .
$$

We define

$$
\|\boldsymbol{\xi}\|=\max _{0 \leq k \leq N}\left\|\boldsymbol{\xi}_{k}\right\|
$$

and place an analogous norm on $\left(\mathbb{R}^{r}\right)^{N}$. Suppose that $L$ has a right inverse $L^{-1_{R}}$.

Let $\varepsilon_{0}$ be a positive number such that

$$
\varepsilon_{0} \leq h_{k} \quad \text { for } k=0, \ldots, N-1 .
$$

The quantity $\sqrt{2} \varepsilon_{0}$ is an a priori upper bound for the shadowing distance $\varepsilon$. Let $\Omega$ be an open convex subset of $\mathbb{R}^{n}$ such that for $k=0, \ldots, N$

$$
\varphi^{t}(\boldsymbol{x}) \in \Omega \quad \text { for } 0 \leq t \leq h_{k}+\varepsilon_{0} \text { and }\left\|\boldsymbol{x}-\boldsymbol{y}_{k}\right\| \leq \sqrt{2} \varepsilon_{0} .
$$

Next let

$$
\begin{gathered}
\mathfrak{M}_{0}=\sup _{\boldsymbol{x} \in \Omega}\|f(\boldsymbol{x})\|, \quad \mathfrak{M}_{1}=\sup _{\boldsymbol{x} \in \Omega}\|D f(\boldsymbol{x})\|, \quad \mathfrak{M}_{2}=\sup _{\boldsymbol{x} \in \Omega}\left\|D^{2} f(\boldsymbol{x})\right\|, \\
\mathfrak{P}_{1}=\sup _{\boldsymbol{x} \in \Omega}\|D g(\boldsymbol{x})\|, \quad \text { and } \quad \mathfrak{P}_{2}=\sup _{\boldsymbol{x} \in \Omega}\left\|D^{2} g(\boldsymbol{x})\right\|
\end{gathered}
$$

and let

$$
\begin{aligned}
\mathfrak{a} & =\inf _{0 \leq k \leq N}\left\|f\left(\boldsymbol{y}_{k}\right)\right\|, \\
\mathfrak{A} & =\sup _{0 \leq k \leq N-1}\left\|Y_{k}\right\|,
\end{aligned}
$$

and

$$
h_{\max }=\max _{0 \leq k \leq N-1} h_{k} .
$$

From (3.2), (3.5), and (3.6) we see that each $D g\left(\boldsymbol{y}_{k}\right) T_{k}$ is a lower triangular $m \times m$ matrix with nonzero diagonal entries. Let

$$
\begin{aligned}
\mathfrak{B} & =\max _{0 \leq k \leq N}\left\|\left[D g\left(\boldsymbol{y}_{k}\right) T_{k}\right]^{-1}\right\| \quad \text { and } \\
\delta_{1} & =\max _{0 \leq k \leq N} \frac{\left\|D g\left(\boldsymbol{y}_{k}\right) f\left(\boldsymbol{y}_{k}\right)\right\|}{\left\|f\left(\boldsymbol{y}_{k}\right)\right\|} .
\end{aligned}
$$

We have now defined the essential quantities that appear in our main theorem below.

Theorem 3.1. Let $\left\{\boldsymbol{y}_{k}\right\}_{k=0}^{N}$ be a $\delta$-pseudo orbit of (1.1) satisfying (3.2) and let

$$
\begin{aligned}
\mathfrak{C} & =\max \left\{\mathfrak{B},(1+\mathfrak{A} \mathfrak{B})\left\|L^{-1_{R}}\right\|, \mathfrak{a}^{-1}(1+\mathfrak{A} \mathfrak{B})\left(1+\mathfrak{A}\left\|L^{-1_{R}}\right\|\right)\right\}, \\
\delta_{K} & =\mathfrak{C}\left(\mathfrak{M}_{1}+2\right) \delta \\
\delta_{\mathcal{H}} & =\left(1+\frac{2 \mathfrak{C}\left(\mathfrak{M}_{0}+\sqrt{2} e^{\mathfrak{M}_{1} h_{\max }}\right)}{1-\delta_{K}}\right) \delta, \quad \text { and } \\
\mathfrak{D} & =\mathfrak{M}_{0} \mathfrak{M}_{1}+2 \sqrt{2} \mathfrak{M}_{1} e^{\mathfrak{M}_{1}\left(h_{\max }+\varepsilon_{0}\right)}+2 \mathfrak{M}_{2}\left(h_{\max }+\varepsilon_{0}\right) e^{2 \mathfrak{M}_{1}\left(h_{\max }+\varepsilon_{0}\right)}
\end{aligned}
$$

If $\delta$ together with $\delta_{K}, \delta_{1}, \delta_{\mathcal{H}}$, and $\varepsilon_{0}$ satisfy the inequalities 
(1) $\delta_{K}<1$,

(2) $\delta_{1}<1$,

(3) $4 \mathfrak{P}_{2} \mathfrak{C}^{2} \delta\left(1-\delta_{K}\right)^{-2} \leq 1$,

(4) $2 \mathfrak{D C} \mathfrak{C}^{2} \delta\left(1-\delta_{K}\right)^{-2} \leq 1$,

(5) $\delta_{\mathcal{H}}<\varepsilon_{0}$, and

(6) $\delta_{\mathcal{H}} \leq\left(1-\delta_{1}\right)\left(\mathfrak{P}_{2} \sqrt{\mathfrak{B}^{2}+2}\right)^{-1}$

then the $\delta$-pseudo orbit $\left\{\boldsymbol{y}_{k}\right\}_{k=0}^{N}$ is $\varepsilon$-shadowed by a true orbit $\left\{\boldsymbol{x}_{k}\right\}_{k=0}^{N}$ with

$$
\varepsilon=\frac{2 \sqrt{2} \mathfrak{C} \delta}{1-\delta_{K}}
$$

Furthermore,

$$
g\left(\boldsymbol{x}_{k}\right)=\mathbf{0} \quad \text { for } k=0, \ldots, N .
$$

\section{Application of the Main Theorem}

The concept of a $\delta$-pseudo orbit gives a natural description of the types of sequences generated by the standard numerical algorithms used to solve initial value problems for ordinary differential equations. As mentioned above, we intend to apply our main theorem to such computer generated sequences. In this section we expand on the description, selection, and computation of the quantities defined in section 3 .

We propose generating the sequences $\left\{\boldsymbol{y}_{k}\right\}_{k=0}^{N}$ and $\left\{Y_{k}\right\}_{k=0}^{N-1}$ starting with $\boldsymbol{y}_{0}$ near the invariant submanifold $\Lambda$ and recursively applying a one step method to the initial value problems

$$
\begin{aligned}
\dot{\boldsymbol{y}} & =f(\boldsymbol{y}), & \boldsymbol{y}(0) & =\boldsymbol{y}_{k}, \\
\dot{Y} & =D f(\boldsymbol{y}) Y, & Y(0) & =I_{n}
\end{aligned}
$$

for $k=0, \ldots, N-1$ obtaining approximations $\overline{\boldsymbol{y}}_{k+1}$ of $\varphi^{h_{k}}\left(\boldsymbol{y}_{k}\right)$ and $Y_{k}$ of $D \varphi^{h_{k}}\left(\boldsymbol{y}_{k}\right)$. Coomes, Koçak, and Palmer [10] used a Taylor series method in their study of finite time shadowing for the Lorenz equations because it allowed them to give a rigorous upper bound for the local error $\delta$ in the computation of $\overline{\boldsymbol{y}}_{k+1}$. Since $\Lambda$ is an invariant set for (1.1) it would seem to be desirable to have $\boldsymbol{y}_{k+1}$ as close to $\Lambda$ as is possible. One option would be to choose a one step method under which $\Lambda$ is invariant and set $\boldsymbol{y}_{k+1}=\overline{\boldsymbol{y}}_{k+1}$. A second option might be to "correct" $\overline{\boldsymbol{y}}_{k+1}$ and choose $\boldsymbol{y}_{k+1}$ so as to not increase $\delta$ significantly. For example, one might choose

$$
\boldsymbol{y}_{k+1}=\overline{\boldsymbol{y}}_{k+1}-D g\left(\overline{\boldsymbol{y}}_{k+1}\right)^{*}\left[D g\left(\overline{\boldsymbol{y}}_{k+1}\right) D g\left(\overline{\boldsymbol{y}}_{k+1}\right)^{*}\right]^{-1} g\left(\overline{\boldsymbol{y}}_{k+1}\right)
$$

provided $D g\left(\overline{\boldsymbol{y}}_{k+1}\right)$ is not rank deficient which is suggested but not implied by (3.2). Another option might be to provide such a correction not at every step, but only if $\overline{\boldsymbol{y}}_{k+1}$ has drifted away from $\Lambda$ by more than some amount on the order of the local error in the one step method for (4.1).

We now turn to the question of what the matrices $A_{k}$, defined in (3.7), represent. To answer this question, for the moment assume that our solution of (4.1) is exact at the $k$ th stage. That is, assume $\boldsymbol{y}_{k+1}=\varphi^{h_{k}}\left(\boldsymbol{y}_{k}\right), Y_{k}=D \varphi^{h_{k}}\left(\boldsymbol{y}_{k}\right)$ and assume $\boldsymbol{y}_{k}$ and $\boldsymbol{y}_{k+1}$ both lie in $\Lambda$. For a smooth manifold $\Sigma$ let $T_{\boldsymbol{p}}(\Sigma)$ denote the tangent space of $\Sigma$ at $\boldsymbol{p}$. Let

$$
\left\langle f\left(\boldsymbol{y}_{k}\right)\right\rangle^{\perp}=\left\{\boldsymbol{x} \in T_{\boldsymbol{y}_{k}}\left(\mathbb{R}^{n}\right):\left\langle\boldsymbol{x}, f\left(\boldsymbol{y}_{k}\right)\right\rangle=0\right\}
$$


where $\langle\cdot, \cdot\rangle$ denotes the usual inner product. With the natural identifications of spaces and operators, notice that the following diagram commutes

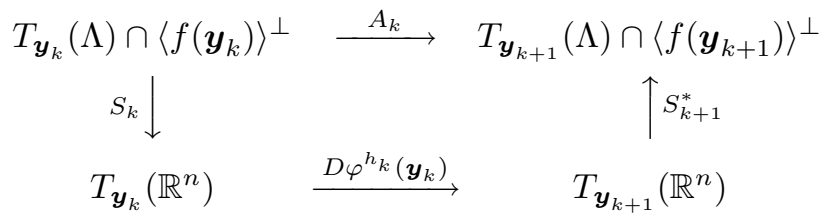

where $S_{k}$ is the natural embedding and $S_{k+1}^{*}$ is orthogonal projection onto the space

$$
T_{\boldsymbol{y}_{k+1}}(\Lambda) \cap\left\langle f\left(\boldsymbol{y}_{k+1}\right)\right\rangle^{\perp} .
$$

That is, in this case $A_{k}$ describes the behavior of the action of the solution of the variational equation on the space of vectors tangent to $\Lambda$ at $\boldsymbol{y}_{k}$ and normal to $f\left(\boldsymbol{y}_{k}\right)$ after projection onto the space of vectors tangent to $\Lambda$ at $\boldsymbol{y}_{k+1}$ and normal to $f\left(\boldsymbol{y}_{k+1}\right)$. We expect $A_{k}$ to describe analogous behavior between $\boldsymbol{x}_{k}$ and $\boldsymbol{x}_{k+1}$ in the case where our quantities are approximate and we are able to obtain a positive conclusion for our main theorem.

Thus far we have insisted that $\mathbf{0}$ be a regular value for $g$. This condition is slightly stronger than is needed in the proof of Theorem 3.1. It suffices to have $\Lambda$, the zero set for $g$, be invariant under the flow for (1.1) as long as (3.2) holds.

The quantity $\left\|L^{-1_{R}}\right\|$, where $L$ is defined in (3.8), figures prominently in the statement of Theorem 3.1, but it may not be obvious how to choose $L^{-1_{R}}$ so that $\left\|L^{-1_{R}}\right\|$ is not too large. As noted above, we expect the nature of $L$ to be governed by the behavior of the restriction of the flow to $\Lambda$. Notice that the matrices $A_{k}$ are upper triangular. By the nature of the QR decomposition, one expects that for most $k$

$$
A_{k}=\left(\begin{array}{cc}
B_{k} & C_{k} \\
0 & D_{k}
\end{array}\right)
$$

where $B_{k}$ is a square upper triangular matrix whose diagonal entries all have absolute value larger than unity and $D_{k}$ is a square upper triangular matrix whose diagonal entries all have absolute value smaller than unity. In addition, in the presence of some aspect of uniform hyperbolicity, one expects the sizes of $B_{k}$ and $D_{k}$ to be independent of $k$. Chow and Palmer [5] and Coomes, Koçak, and Palmer [10] show how to exploit this structure to obtain a suitable right inverse for $L$ and compute a bound for its norm. Thus if the flow for (1.1) restricted to $\Lambda$ exhibits some aspect of uniform hyperbolicity, we expect to be able to find a similar bound.

The convex set $\Omega$ and the estimate $\varepsilon_{0}$ are chosen a priori. The set $\Omega$ must contain a neighborhood of each of the vectors $\boldsymbol{y}_{k}$ as specified in (3.10), but should be chosen as small as possible to keep $\mathfrak{M}_{0}, \mathfrak{M}_{1}, \mathfrak{M}_{2}, \mathfrak{P}_{1}$, and $\mathfrak{P}_{2}$ from being too large. Hence, the estimate $\varepsilon_{0}$ should be chosen small enough to keep (3.10) from being too onerous and $\mathfrak{D}$ from being too large, but not so small that hypothesis (5) of Theorem 3.1 cannot be satisfied. A reasonable compromise might be to choose $\varepsilon_{0}$ as large as possible yet small enough to still have shadowing of the $\delta$-pseudo orbit within $\sqrt{2} \varepsilon_{0}$ be interesting.

We comment on some of the other quantities described in section 3. To insure that $\mathfrak{a}$ is not too small, our $\delta$-pseudo orbit must not come too close to a fixed point of $f$. If the step sizes $h_{k}$ are sufficiently small, the matrices $Y_{k}$ will be close to the identity, but hopefully far enough from the identity for the QR decomposition to effectively isolate local expanding and contracting directions. The quantity $\mathfrak{B}$ 
will not be too large if the matrix in (3.2) is never close to a matrix which is rank deficient. And if $g$ is a first integral for (1.1), we have $\delta_{1}=0$; otherwise we assume that near $\Lambda$ the vector field $f$ is nearly orthogonal to the gradients of the components of $g$.

Lastly, we mention a way to possibly weaken hypothesis (4) of Theorem 3.1. If we let $\varepsilon_{\mathcal{G}}$ be as in (5.20),

$$
\overline{\mathfrak{M}}_{0}=\max _{0 \leq k \leq N}\left\|f\left(\boldsymbol{y}_{k}\right)\right\|, \quad \text { and } \quad \overline{\mathfrak{M}}_{1}=\max _{0 \leq k \leq N}\left\|D f\left(\boldsymbol{y}_{k}\right)\right\|,
$$

then we may replace $\mathfrak{D}$ in hypothesis (4) with

$$
\begin{aligned}
\overline{\mathfrak{D}}=\left(\overline{\mathfrak{M}}_{0}+\mathfrak{M}_{1} \delta_{\mathcal{H}}\right)\left(\overline{\mathfrak{M}}_{1}+\mathfrak{M}_{2} \delta_{\mathcal{H}}\right)+2 \sqrt{2}\left(\overline{\mathfrak{M}}_{1}+\mathfrak{M}_{2} \delta_{\mathcal{H}}\right) e^{\mathfrak{M}_{1}\left(h_{\max }+\varepsilon_{\mathcal{G}}\right)} \\
+2 \mathfrak{M}_{2}\left(h_{\max }+\varepsilon_{\mathcal{G}}\right) e^{2 \mathfrak{M}_{1}\left(h_{\max }+\varepsilon_{\mathcal{G}}\right)} .
\end{aligned}
$$

See (5.1), (5.10), (5.11), (5.24), (5.25), and (5.36) in the proof of the main theorem.

\section{Proof of the Main Theorem}

We assume that $\left\{\boldsymbol{y}_{k}\right\}_{k=0}^{N}$ is a $\delta$-pseudo orbit for (1.1) and that all of the hypotheses of Theorem 3.1 are satisfied by the various quantities defined in section 3 . We first recast the problem of finding an appropriate true orbit of (1.1) as that of finding an appropriate root of a particular function. The $\delta$-pseudo orbit $\left\{\boldsymbol{y}_{k}\right\}_{k=0}^{N}$ will correspond to a point where the norm of the function is small and we will prove the existence of a nearby root. We will complete the proof by showing that the root of this function does indeed correspond to a true orbit of (1.1). We start with the following variation of the Implicit Function Theorem.

Lemma 5.1. Let $\mathbb{X}$ and $\mathbb{Y}$ be finite dimensional normed vector spaces over $\mathbb{R}$, let $\Gamma_{\mathcal{G}}$ be an open subset of $\mathbb{X}$, and let $\mathcal{G}: \Gamma_{\mathcal{G}} \rightarrow \mathbb{Y}$ be a $C^{2}$ function. Suppose that there exists $\boldsymbol{u}_{0}$ in $\Gamma_{\mathcal{G}}$ such that the derivative $D \mathcal{G}\left(\boldsymbol{u}_{0}\right)$ has a right inverse $K$. Let

$$
\varepsilon_{\mathcal{G}}=2\|K\|\left\|\mathcal{G}\left(\boldsymbol{u}_{0}\right)\right\|
$$

and suppose that the closed ball of radius $\varepsilon_{\mathcal{G}}$ is contained in $\Gamma_{\mathcal{G}}$. Let

$$
\mathfrak{F}=\sup \left\{\left\|D^{2} \mathcal{G}(\boldsymbol{u})\right\|:\left\|\boldsymbol{u}-\boldsymbol{u}_{0}\right\| \leq \varepsilon_{\mathcal{G}}\right\}
$$

and suppose further that

$$
2 \mathfrak{F}\|K\|^{2}\left\|\mathcal{G}\left(\boldsymbol{u}_{0}\right)\right\| \leq 1
$$

Then there is a solution $\overline{\boldsymbol{u}}$ of the equation

$$
\mathcal{G}(\overline{\boldsymbol{u}})=\mathbf{0}
$$

satisfying $\left\|\overline{\boldsymbol{u}}-\boldsymbol{u}_{0}\right\| \leq \varepsilon_{\mathcal{G}}$.

This lemma is Lemma 4.1 in [7] which contains a proof based on the Brouwer Fixed Point Theorem.

We now construct the function to which we will apply Lemma 5.1. Let $\mathbb{X}=$ $\mathbb{R}^{N} \times\left(\mathbb{R}^{m}\right)^{N+1} \times\left(\mathbb{R}^{r}\right)^{N+1}$ and let $\mathbb{Y}=\left(\mathbb{R}^{m}\right)^{N+1} \times\left(\mathbb{R}^{r}\right)^{N} \times \mathbb{R}^{N}$. Denote

$$
\begin{aligned}
& \boldsymbol{u}=\left(\left\{s_{k}\right\}_{k=0}^{N-1},\left\{\boldsymbol{v}_{k}\right\}_{k=0}^{N},\left\{\boldsymbol{w}_{k}\right\}_{k=0}^{N}\right) \in \mathbb{X} \quad \text { and } \\
& \boldsymbol{z}=\left(\left\{\boldsymbol{\psi}_{k}\right\}_{k=0}^{N},\left\{\boldsymbol{\omega}_{k}\right\}_{k=0}^{N-1},\left\{\alpha_{k}\right\}_{k=0}^{N-1}\right) \in \mathbb{Y} .
\end{aligned}
$$


Next we define

$$
\begin{aligned}
& \|\boldsymbol{u}\|=\max \left\{\max _{0 \leq k \leq N-1}\left|s_{k}\right|, \max _{0 \leq k \leq N}\left\|\boldsymbol{v}_{k}\right\|, \max _{0 \leq k \leq N}\left\|\boldsymbol{w}_{k}\right\|\right\}, \quad \text { and } \\
& \|\boldsymbol{z}\|=\max \left\{\max _{0 \leq k \leq N}\left\|\boldsymbol{\psi}_{k}\right\|, \max _{0 \leq k \leq N-1}\left\|\boldsymbol{\omega}_{k}\right\|, \max _{0 \leq k \leq N-1}\left|\alpha_{k}\right|\right\} .
\end{aligned}
$$

Let

$$
\boldsymbol{u}_{0}=\left(\left\{h_{k}\right\}_{k=0}^{N-1},\{\mathbf{0}\}_{k=0}^{N},\{\mathbf{0}\}_{k=0}^{N}\right) \in \mathbb{X}
$$

and denote

$$
\boldsymbol{f}_{k}=f\left(\boldsymbol{y}_{k}\right) /\left\|f\left(\boldsymbol{y}_{k}\right)\right\| \quad \text { for } k=0, \ldots, N .
$$

Let $\Gamma_{\mathcal{G}}$ be the open ball in $\mathbb{X}$ about $\boldsymbol{u}_{0}$ of radius $\varepsilon_{0}$. Then by (3.9) and (3.10) we see that if $\boldsymbol{u} \in \Gamma_{\mathcal{G}}$ then $\varphi^{s_{k}}\left(\boldsymbol{y}_{k}+T_{k} \boldsymbol{v}_{k}+S_{k} \boldsymbol{w}_{k}\right)$ is defined and lies in $\Omega$. We define $\mathcal{G}: \Gamma_{\mathcal{G}} \rightarrow \mathbb{Y}$ by

$$
\mathcal{G}(\boldsymbol{u})=\left(\mathcal{G}_{1}(\boldsymbol{u}), \mathcal{G}_{2}(\boldsymbol{u}), \mathcal{G}_{3}(\boldsymbol{u})\right)
$$

where for $k=0, \ldots, N$

$$
\left[\mathcal{G}_{1}(\boldsymbol{u})\right]_{k}=g\left(\boldsymbol{y}_{k}+T_{k} \boldsymbol{v}_{k}+S_{k} \boldsymbol{w}_{k}\right),
$$

and for $k=0, \ldots, N-1$

$$
\begin{aligned}
& {\left[\mathcal{G}_{2}(\boldsymbol{u})\right]_{k}=\boldsymbol{w}_{k+1}+S_{k+1}^{*}\left(\boldsymbol{y}_{k+1}-\varphi^{s_{k}}\left(\boldsymbol{y}_{k}+T_{k} \boldsymbol{v}_{k}+S_{k} \boldsymbol{w}_{k}\right)\right), \quad \text { and }} \\
& {\left[\mathcal{G}_{3}(\boldsymbol{u})\right]_{k}=\boldsymbol{f}_{k+1}^{*}\left(\boldsymbol{y}_{k+1}-\varphi^{s_{k}}\left(\boldsymbol{y}_{k}+T_{k} \boldsymbol{v}_{k}+S_{k} \boldsymbol{w}_{k}\right)\right) .}
\end{aligned}
$$

By (3.1) and (3.2) it follows that

$$
\left\|\mathcal{G}\left(\boldsymbol{u}_{0}\right)\right\| \leq \delta
$$

Notice that for

$$
\boldsymbol{u}_{1}=\left(\left\{\sigma_{k}\right\}_{k=0}^{N-1},\left\{\boldsymbol{\zeta}_{k}\right\}_{k=0}^{N},\left\{\boldsymbol{\eta}_{k}\right\}_{k=0}^{N}\right) \in \mathbb{X}
$$

we have

$$
D \mathcal{G}\left(\boldsymbol{u}_{0}\right) \boldsymbol{u}_{1}=\left(D \mathcal{G}_{1}\left(\boldsymbol{u}_{0}\right) \boldsymbol{u}_{1}, D \mathcal{G}_{2}\left(\boldsymbol{u}_{0}\right) \boldsymbol{u}_{1}, D \mathcal{G}_{3}\left(\boldsymbol{u}_{0}\right) \boldsymbol{u}_{1}\right) .
$$

By (3.5) and (3.6) it follows that $D g\left(\boldsymbol{y}_{k}\right) S_{k}=0$ for $k=0, \ldots, N$. Hence for $k=$ $0, \ldots, N$

$$
\left[D \mathcal{G}_{1}\left(\boldsymbol{u}_{0}\right) \boldsymbol{u}_{1}\right]_{k}=D g\left(\boldsymbol{y}_{k}\right) T_{k} \boldsymbol{\zeta}_{k}
$$

and for $k=0, \ldots, N-1$

$$
\left[D \mathcal{G}_{2}\left(\boldsymbol{u}_{0}\right) \boldsymbol{u}_{1}\right]_{k}=\boldsymbol{\eta}_{k+1}-S_{k+1}^{*}\left(\sigma_{k} f\left(\varphi^{h_{k}}\left(\boldsymbol{y}_{k}\right)\right)+D \varphi^{h_{k}}\left(\boldsymbol{y}_{k}\right)\left(T_{k} \boldsymbol{\zeta}_{k}+S_{k} \boldsymbol{\eta}_{k}\right)\right)
$$

and

$$
\left[D \mathcal{G}_{3}\left(\boldsymbol{u}_{0}\right) \boldsymbol{u}_{1}\right]_{k}=-\boldsymbol{f}_{k+1}^{*}\left(\sigma_{k} f\left(\varphi^{h_{k}}\left(\boldsymbol{y}_{k}\right)\right)+D \varphi^{h_{k}}\left(\boldsymbol{y}_{k}\right)\left(T_{k} \boldsymbol{\zeta}_{k}+S_{k} \boldsymbol{\eta}_{k}\right)\right) .
$$

Furthermore, notice that for

$$
\boldsymbol{u}_{2}=\left(\left\{\tau_{k}\right\}_{k=0}^{N-1},\left\{\boldsymbol{\nu}_{k}\right\}_{k=0}^{N},\left\{\boldsymbol{\xi}_{k}\right\}_{k=0}^{N}\right)
$$

we have

$$
D^{2} \mathcal{G}(\boldsymbol{u}) \boldsymbol{u}_{1} \boldsymbol{u}_{2}=\left(D^{2} \mathcal{G}_{1}(\boldsymbol{u}) \boldsymbol{u}_{1} \boldsymbol{u}_{2}, D^{2} \mathcal{G}_{2}(\boldsymbol{u}) \boldsymbol{u}_{1} \boldsymbol{u}_{2}, D^{2} \mathcal{G}_{3}(\boldsymbol{u}) \boldsymbol{u}_{1} \boldsymbol{u}_{2}\right)
$$

where for $k=0, \ldots, N$

$$
\left[D^{2} \mathcal{G}_{1}(\boldsymbol{u}) \boldsymbol{u}_{1} \boldsymbol{u}_{2}\right]_{k}=D^{2} g\left(\boldsymbol{y}_{k}+T_{k} \boldsymbol{v}_{k}+S_{k} \boldsymbol{w}_{k}\right)\left(T_{k} \boldsymbol{\zeta}_{k}+S_{k} \boldsymbol{\eta}\right)\left(T_{k} \boldsymbol{\nu}_{k}+S_{k} \boldsymbol{\xi}\right)
$$


and for $k=0, \ldots, N-1$

$$
\begin{aligned}
{\left[D^{2} \mathcal{G}_{2}(\boldsymbol{u}) \boldsymbol{u}_{1} \boldsymbol{u}_{2}\right]_{k} } & \\
= & -S_{k+1}^{*}\left(\sigma_{k} \tau_{k} D f\left(\varphi^{s_{k}}\left(\boldsymbol{y}_{k}+T_{k} \boldsymbol{v}_{k}+S_{k} \boldsymbol{w}_{k}\right)\right) f\left(\varphi^{s_{k}}\left(\boldsymbol{y}_{k}+T_{k} \boldsymbol{v}_{k}+S_{k} \boldsymbol{w}_{k}\right)\right)\right. \\
& +\sigma_{k} D f\left(\varphi^{s_{k}}\left(\boldsymbol{y}_{k}+T_{k} \boldsymbol{v}_{k}+S_{k} \boldsymbol{w}_{k}\right)\right) D \varphi^{s_{k}}\left(\boldsymbol{y}_{k}+T_{k} \boldsymbol{v}_{k}+S_{k} \boldsymbol{w}_{k}\right)\left(T_{k} \boldsymbol{\nu}_{k}+S_{k} \boldsymbol{\xi}_{k}\right) \\
& +\tau_{k} D f\left(\varphi^{s_{k}}\left(\boldsymbol{y}_{k}+T_{k} \boldsymbol{v}_{k}+S_{k} \boldsymbol{w}_{k}\right)\right) D \varphi^{s_{k}}\left(\boldsymbol{y}_{k}+T_{k} \boldsymbol{v}_{k}+S_{k} \boldsymbol{w}_{k}\right)\left(T_{k} \boldsymbol{\zeta}_{k}+S_{k} \boldsymbol{\eta}_{k}\right) \\
& \left.+D^{2} \varphi^{s_{k}}\left(\boldsymbol{y}_{k}+T_{k} \boldsymbol{v}_{k}+S_{k} \boldsymbol{w}_{k}\right)\left(T_{k} \boldsymbol{\zeta}_{k}+S_{k} \boldsymbol{\eta}_{k}\right)\left(T_{k} \boldsymbol{\nu}_{k}+S_{k} \boldsymbol{\xi}_{k}\right)\right)
\end{aligned}
$$

and

$$
\begin{aligned}
{\left[D^{2} \mathcal{G}_{3}(\boldsymbol{u}) \boldsymbol{u}_{1} \boldsymbol{u}_{2}\right]_{k} } & \\
= & -\boldsymbol{f}_{k+1}^{*}\left(\sigma_{k} \tau_{k} D f\left(\varphi^{s_{k}}\left(\boldsymbol{y}_{k}+T_{k} \boldsymbol{v}_{k}+S_{k} \boldsymbol{w}_{k}\right)\right) f\left(\varphi^{s_{k}}\left(\boldsymbol{y}_{k}+T_{k} \boldsymbol{v}_{k}+S_{k} \boldsymbol{w}_{k}\right)\right)\right. \\
& +\sigma_{k} D f\left(\varphi^{s_{k}}\left(\boldsymbol{y}_{k}+T_{k} \boldsymbol{v}_{k}+S_{k} \boldsymbol{w}_{k}\right)\right) D \varphi^{s_{k}}\left(\boldsymbol{y}_{k}+T_{k} \boldsymbol{v}_{k}+S_{k} \boldsymbol{w}_{k}\right)\left(T_{k} \boldsymbol{\nu}_{k}+S_{k} \boldsymbol{\xi}_{k}\right) \\
& +\tau_{k} D f\left(\varphi^{s_{k}}\left(\boldsymbol{y}_{k}+T_{k} \boldsymbol{v}_{k}+S_{k} \boldsymbol{w}_{k}\right)\right) D \varphi^{s_{k}}\left(\boldsymbol{y}_{k}+T_{k} \boldsymbol{v}_{k}+S_{k} \boldsymbol{w}_{k}\right)\left(T_{k} \boldsymbol{\zeta}_{k}+S_{k} \boldsymbol{\eta}_{k}\right) \\
& \left.+D^{2} \varphi^{s_{k}}\left(\boldsymbol{y}_{k}+T_{k} \boldsymbol{v}_{k}+S_{k} \boldsymbol{w}_{k}\right)\left(T_{k} \boldsymbol{\zeta}_{k}+S_{k} \boldsymbol{\eta}_{k}\right)\left(T_{k} \boldsymbol{\nu}_{k}+S_{k} \boldsymbol{\xi}_{k}\right)\right) .
\end{aligned}
$$

Lemma 5.2. The linear map $D \mathcal{G}\left(\boldsymbol{u}_{0}\right)$ has a right inverse $K$ and

$$
\|K\| \leq \frac{\mathfrak{C}}{1-\mathfrak{C}\left(\mathfrak{M}_{1}+2\right) \delta}=\frac{\mathfrak{C}}{1-\delta_{K}} .
$$

Proof. Approximate $D \mathcal{G}\left(\boldsymbol{u}_{0}\right)$ by the linear operator $U: \mathbb{X} \rightarrow \mathbb{Y}$ given by $U=$ $\left(U_{1}, U_{2}, U_{3}\right)$ where $U_{1}=D \mathcal{G}_{1}\left(\boldsymbol{u}_{0}\right)$ and for $k=0, \ldots, N-1$

$$
\begin{aligned}
& {\left[U_{2} \boldsymbol{u}_{1}\right]_{k}=\boldsymbol{\eta}_{k+1}-S_{k+1}^{*} Y_{k} T_{k} \boldsymbol{\zeta}_{k}-A_{k} \boldsymbol{\eta}_{k}, \quad \text { and }} \\
& {\left[U_{3} \boldsymbol{u}_{1}\right]_{k}=-\boldsymbol{f}_{k+1}^{*}\left(\sigma_{k} f\left(\boldsymbol{y}_{k+1}\right)+Y_{k}\left(T_{k} \boldsymbol{\zeta}_{k}+S_{k} \boldsymbol{\eta}_{k}\right)\right) .}
\end{aligned}
$$

Notice that by (3.1), (3.3), (3.5), (3.6), (3.7), (3.11), (5.7), (5.8), and (5.13) we have

$$
\left\|D \mathcal{G}\left(\boldsymbol{u}_{0}\right)-U\right\| \leq\left(\mathfrak{M}_{1}+2\right) \delta .
$$

Furthermore, one can construct a right inverse for $U$ using the right inverse $L^{-1_{R}}$ for the operator $L$ defined by (3.8). The construction follows: Let

$$
\boldsymbol{z}=\left(\left\{\boldsymbol{\psi}_{k}\right\}_{k=0}^{N},\left\{\boldsymbol{\omega}_{k}\right\}_{k=0}^{N-1},\left\{\alpha_{k}\right\}_{k=0}^{N-1}\right) \in \mathbb{Y} .
$$

From the comments preceding (3.15) we see that each $D g\left(\boldsymbol{y}_{k}\right) T_{k}$ is invertible. Thus set

$$
\boldsymbol{\zeta}_{k}=\left[D g\left(\boldsymbol{y}_{k}\right) T_{k}\right]^{-1} \boldsymbol{\psi}_{k} \quad \text { for } k=0, \ldots, N
$$

Next set

$$
\left\{\boldsymbol{\eta}_{k}\right\}_{k=0}^{N}=L^{-1_{R}}\left(\left\{S_{k+1}^{*} Y_{k} T_{k} \boldsymbol{\zeta}_{k}+\boldsymbol{\omega}_{k}\right\}_{k=0}^{N-1}\right)
$$

and finally set

$$
\sigma_{k}=\frac{-\alpha_{k}-\boldsymbol{f}_{k+1}^{*} Y_{k}\left(T_{k} \boldsymbol{\zeta}_{k}+S_{k} \boldsymbol{\eta}_{k}\right)}{\left\|f\left(\boldsymbol{y}_{k}\right)\right\|} \quad \text { for } k=0, \ldots, N-1 .
$$

Notice that we have

$$
U\left(\left\{\sigma_{k}\right\}_{k=0}^{N-1},\left\{\boldsymbol{\zeta}_{k}\right\}_{k=0}^{N},\left\{\boldsymbol{\eta}_{k}\right\}_{k=0}^{N}\right)=\boldsymbol{z} .
$$


Thus we have defined a right inverse $U^{-1_{R}}$ for $U$. By (3.13), (3.14), (3.15), (5.15), (5.16), and (5.17) we see that

$$
\left\|U^{-1_{R}}\right\| \leq \max \left\{\mathfrak{B},(1+\mathfrak{A} \mathfrak{B})\left\|L^{-1_{R}}\right\|, \mathfrak{a}^{-1}(1+\mathfrak{A} \mathfrak{B})\left(1+\mathfrak{A}\left\|L^{-1_{R}}\right\|\right)\right\}=\mathfrak{C} .
$$

Hence by hypothesis (1) of Theorem 3.1, (5.14), and (5.18) we see that

$$
\left\|I_{\mathbb{Y}}-D \mathcal{G}\left(\boldsymbol{u}_{0}\right) U^{-1_{R}}\right\| \leq\left\|U-D \mathcal{G}\left(\boldsymbol{u}_{0}\right)\right\|\left\|U^{-1_{R}}\right\| \leq\left(\mathfrak{M}_{1}+2\right) \mathfrak{C} \delta=\delta_{K}<1 .
$$

Thus the linear operator $D \mathcal{G}\left(\boldsymbol{u}_{0}\right) U^{-1_{R}}$ is invertible and

$$
\left\|\left(D \mathcal{G}\left(\boldsymbol{u}_{0}\right) U^{-1_{R}}\right)^{-1}\right\| \leq \frac{1}{1-\mathfrak{C}\left(\mathfrak{M}_{1}+2\right) \delta} .
$$

Thus the linear operator

$$
K=U^{-1_{R}}\left(D \mathcal{G}\left(\boldsymbol{u}_{0}\right) U^{-1_{R}}\right)^{-1}
$$

is a right inverse for $D \mathcal{G}\left(\boldsymbol{u}_{0}\right)$ and (5.12) follows from (5.18) and (5.19).

Let

$$
\varepsilon_{\mathcal{G}}=\frac{2 \mathfrak{C} \delta}{1-\mathfrak{C}\left(\mathfrak{M}_{1}+2\right) \delta}=\frac{2 \mathfrak{C} \delta}{1-\delta_{K}} .
$$

Then by hypothesis $(5)$ of Theorem $3.1, \varepsilon_{\mathcal{G}}<\varepsilon_{0}$ and hence the closed ball about $\boldsymbol{u}_{0}$ of radius $\varepsilon_{\mathcal{G}}$ lies in $\Gamma_{\mathcal{G}}$.

Lemma 5.3. The local bound $\mathfrak{F}$ for $\left\|D^{2} \mathcal{G}\right\|$ defined in (5.1) satisfies

$$
2 \mathfrak{F}\|K\|^{2}\left\|\mathcal{G}\left(\boldsymbol{u}_{0}\right)\right\| \leq 1 .
$$

Proof. To compute $\mathfrak{F}$ notice that for $\boldsymbol{u}$ in $\Gamma_{\mathcal{G}}$ we have

$$
\left\|D^{2} \mathcal{G}(\boldsymbol{u})\right\|=\max \left\{\left\|D^{2} \mathcal{G}_{1}(\boldsymbol{u})\right\|,\left\|D^{2} \mathcal{G}_{2}(\boldsymbol{u})\right\|,\left\|D^{2} \mathcal{G}_{3}(\boldsymbol{u})\right\|\right\} .
$$

From (3.10), (3.12) and (5.9) we see that

$$
\left\|D^{2} \mathcal{G}_{1}(\boldsymbol{u})\right\| \leq 2 \mathfrak{P}_{2} .
$$

We now compute bounds for norms of $D^{2} \mathcal{G}_{2}(\boldsymbol{u})$ and $D^{2} \mathcal{G}_{3}(\boldsymbol{u})$. For $k=0, \ldots, N-$ $1,\left\|\boldsymbol{x}-\boldsymbol{y}_{k}\right\| \leq \varepsilon_{0}$ and $0 \leq t \leq h_{k}+\varepsilon_{0}$ it follows from (3.9), (3.10), (3.11), Gronwall's Lemma, and the variation of constants formula (see [7] for details) that

$$
\left\|D \varphi^{t}(\boldsymbol{x})\right\| \leq e^{\mathfrak{M}_{1} t} \quad \text { and } \quad\left\|D^{2} \varphi^{t}(\boldsymbol{x})\right\| \leq \mathfrak{M}_{2} t e^{2 \mathfrak{M}_{1} t} .
$$

Thus from (3.9), (3.10), (5.10), (5.11), and (5.24) it follows that $\left\|D^{2} \mathcal{G}_{2}(\boldsymbol{u})\right\|$ and $\left\|D^{2} \mathcal{G}_{3}(\boldsymbol{u})\right\|$ are both bounded by

$$
\mathfrak{D}=\mathfrak{M}_{0} \mathfrak{M}_{1}+2 \sqrt{2} \mathfrak{M}_{1} e^{\mathfrak{M}_{1}\left(h_{\max }+\varepsilon_{0}\right)}+2 \mathfrak{M}_{2}\left(h_{\max }+\varepsilon_{0}\right) e^{2 \mathfrak{M}_{1}\left(h_{\max }+\varepsilon_{0}\right)} .
$$

From hypotheses (3) and (4), of Theorem 3.1, (5.6), Lemma 5.2, (5.22), (5.23) and (5.25) we see that (5.21) holds.

The existence of a root of $\mathcal{G}$ nearby to $\boldsymbol{u}_{0}$ now follows immediately. But before we give the proof of our main theorem we need a final pair of lemmas which we will use to show that the root corresponds to an appropriate true orbit of (1.1). The first of these lemmas is a variation of the Inverse Function Theorem. 
Lemma 5.4. Let $\mathbb{E}$ and $\mathbb{F}$ be Banach spaces, let $\Gamma_{\mathcal{H}}$ be an open subset of $\mathbb{E}$ and suppose $\mathcal{H}: \Gamma_{\mathcal{H}} \rightarrow \mathbb{F}$ is class $C^{2}$. Suppose that $\boldsymbol{p}_{0}$ lies in $\Gamma_{\mathcal{H}}$ and $D \mathcal{H}\left(\boldsymbol{p}_{0}\right)$ has a left inverse $\left[D \mathcal{H}\left(\boldsymbol{p}_{0}\right)\right]^{-1_{L}}$. Suppose further that $\varepsilon_{\mathcal{H}}>0$, the closed ball $\bar{B}\left(\boldsymbol{p}_{0}, \varepsilon_{\mathcal{H}}\right)$ of radius $\varepsilon_{\mathcal{H}}$ about $\boldsymbol{p}_{0}$ is contained in $\Gamma_{\mathcal{H}}$, and

$$
\left\|D^{2} \mathcal{H}(\boldsymbol{p})\right\| \leq \mathfrak{H} \quad \text { for all } \boldsymbol{p} \in \bar{B}\left(\boldsymbol{p}_{0}, \varepsilon_{\mathcal{H}}\right)
$$

If

$$
\varepsilon_{\mathcal{H}}<\frac{1}{\mathfrak{H}\left\|\left[D \mathcal{H}\left(\boldsymbol{p}_{0}\right)\right]^{-1_{L}}\right\|}
$$

then $\left.\mathcal{H}\right|_{\bar{B}\left(\boldsymbol{p}_{0}, \varepsilon_{\mathcal{H}}\right)}$ is injective.

Proof. Suppose $\boldsymbol{p}$ and $\boldsymbol{q}$ lie in $\bar{B}\left(\boldsymbol{p}_{0}, \varepsilon_{\mathcal{H}}\right)$ and define the $C^{2}$ function $\boldsymbol{\theta}:[0,1] \rightarrow \mathbb{F}$ by

$$
\boldsymbol{\theta}(t)=\left[D \mathcal{H}\left(\boldsymbol{p}_{0}\right)\right]^{-1_{L}}\{\mathcal{H}(t \boldsymbol{p}+(1-t) \boldsymbol{q})-\mathcal{H}(\boldsymbol{p})\}-t(\boldsymbol{p}-\boldsymbol{q}) .
$$

Hence

$$
\begin{aligned}
\|\boldsymbol{\theta}(1)-\boldsymbol{\theta}(0)\| & \leq \int_{0}^{1}\left\|\boldsymbol{\theta}^{\prime}(t)\right\| d t \\
& =\int_{0}^{1}\left\|\left[D \mathcal{H}\left(\boldsymbol{p}_{0}\right)\right]^{-1_{L}}\left\{D \mathcal{H}(t \boldsymbol{p}+(1-t) \boldsymbol{q})-D \mathcal{H}\left(\boldsymbol{p}_{0}\right)\right\}(\boldsymbol{p}-\boldsymbol{q})\right\| d t \\
& \leq\left\|\left[D \mathcal{H}\left(\boldsymbol{p}_{0}\right)\right]^{-1_{L}}\right\| \mathfrak{H} \varepsilon_{\mathcal{H}}\|\boldsymbol{p}-\boldsymbol{q}\| .
\end{aligned}
$$

Since $\boldsymbol{\theta}(0)=\mathbf{0}$ and

$$
\boldsymbol{\theta}(1)=\left[D \mathcal{H}\left(\boldsymbol{p}_{0}\right)\right]^{-1_{L}}(\mathcal{H}(\boldsymbol{p})-\mathcal{H}(\boldsymbol{q}))-(\boldsymbol{p}-\boldsymbol{q}),
$$

it follows from the triangle inequality, (5.27) and (5.28) that

$$
\|\boldsymbol{p}-\boldsymbol{q}\|\left(1-\left\|\left[D \mathcal{H}\left(\boldsymbol{p}_{0}\right)\right]^{-1_{L}}\right\| \mathfrak{H} \varepsilon_{\mathcal{H}}\right) \leq\left\|\left[D \mathcal{H}\left(\boldsymbol{p}_{0}\right)\right]^{-1_{L}}(\mathcal{H}(\boldsymbol{p})-\mathcal{H}(\boldsymbol{q}))\right\| .
$$

Thus from (5.26) and (5.29) it follows that

$$
\mathcal{H}(\boldsymbol{p}) \neq \mathcal{H}(\boldsymbol{q}) \quad \text { if } \boldsymbol{p} \neq \boldsymbol{q} .
$$

Hence $\left.\mathcal{H}\right|_{\bar{B}\left(\boldsymbol{p}_{0}, \varepsilon_{\mathcal{H}}\right)}$ is injective.

We have the following application of the previous lemma.

Lemma 5.5. Define $\mathcal{H}_{k}: \mathbb{R}^{n} \rightarrow \mathbb{R}^{m} \times \mathbb{R}^{r} \times \mathbb{R}$ by

$$
\mathcal{H}_{k}(\boldsymbol{x})=\left(g(\boldsymbol{x}), S_{k}^{*} \boldsymbol{x}, \boldsymbol{f}_{k}^{*} \boldsymbol{x}\right)
$$

and choose $\varepsilon_{\mathcal{H}}>0$ such that

$$
\varepsilon_{\mathcal{H}}<\frac{1-\delta_{1}}{\mathfrak{P}_{2} \sqrt{\mathfrak{B}^{2}+2}} \quad \text { and } \quad \varepsilon_{\mathcal{H}} \leq \varepsilon_{0} .
$$

Then $\left.\mathcal{H}_{k}\right|_{\bar{B}\left(\boldsymbol{y}_{k}, \varepsilon_{\mathcal{H}}\right)}$ is injective. 
Proof. We take the norm of a vector in $\mathbb{R}^{m} \times \mathbb{R}^{r} \times \mathbb{R}$ to be the maximum of the norms of its three components. For $\boldsymbol{p}, \boldsymbol{p}_{1}$, and $\boldsymbol{p}_{2}$ in $\mathbb{R}^{n}$ we have

$$
\begin{aligned}
D \mathcal{H}_{k}\left(\boldsymbol{y}_{k}\right) \boldsymbol{p}_{1} & =\left(D g\left(\boldsymbol{y}_{k}\right) \boldsymbol{p}_{1}, S_{k}^{*} \boldsymbol{p}_{1}, \boldsymbol{f}_{k}^{*} \boldsymbol{p}_{1}\right) \quad \text { and } \\
D^{2} \mathcal{H}_{k}(\boldsymbol{p}) \boldsymbol{p}_{1} \boldsymbol{p}_{2} & =\left(D^{2} g(\boldsymbol{p}) \boldsymbol{p}_{1} \boldsymbol{p}_{2}, \mathbf{0}, 0\right) .
\end{aligned}
$$

Let $(\boldsymbol{\zeta}, \boldsymbol{\eta}, \sigma)$ be in $\mathbb{R}^{m} \times \mathbb{R}^{r} \times \mathbb{R}$ and define the linear operator $V: \mathbb{R}^{m} \times \mathbb{R}^{r} \times \mathbb{R} \rightarrow \mathbb{R}^{n}$ by

$$
V(\boldsymbol{\zeta}, \boldsymbol{\eta}, \sigma)=T_{k}\left[D g\left(\boldsymbol{y}_{k}\right) T_{k}\right]^{-1} \boldsymbol{\zeta}+S_{k} \boldsymbol{\eta}+\boldsymbol{f}_{k} \sigma .
$$

From (3.5) and (3.6) we see that

$$
D \mathcal{H}_{k}\left(\boldsymbol{y}_{k}\right) V(\boldsymbol{\zeta}, \boldsymbol{\eta}, \sigma)=\left(\boldsymbol{\zeta}+D g\left(\boldsymbol{y}_{k}\right) \boldsymbol{f}_{k} \sigma, \boldsymbol{\eta}, \sigma\right)
$$

and thus by (3.16)

$$
\left\|I_{\mathbb{R}^{m} \times \mathbb{R}^{r} \times \mathbb{R}}-D \mathcal{H}_{k}\left(\boldsymbol{y}_{k}\right) V\right\| \leq \delta_{1} .
$$

By hypothesis (2) of Theorem 3.1, the linear operator $D \mathcal{H}_{k}\left(\boldsymbol{y}_{k}\right) V$ is invertible with

$$
\left\|\left[D \mathcal{H}_{k}\left(\boldsymbol{y}_{k}\right) V\right]^{-1}\right\| \leq \frac{1}{1-\delta_{1}}
$$

In addition, from (3.15) we see that

$$
\|V\| \leq \sqrt{\mathfrak{B}^{2}+2}
$$

and hence $D \mathcal{H}_{k}\left(\boldsymbol{y}_{k}\right)$ has inverse $V\left[D \mathcal{H}_{k}\left(\boldsymbol{y}_{k}\right) V\right]^{-1}$ which implies

$$
\left\|\left[D \mathcal{H}_{k}\left(\boldsymbol{y}_{k}\right)\right]^{-1}\right\| \leq \frac{\sqrt{\mathfrak{B}^{2}+2}}{1-\delta_{1}} .
$$

By (3.10), (3.12), (5.30) and (5.31) it follows that

$$
\left\|D^{2} \mathcal{H}_{k}(\boldsymbol{p})\right\|=\left\|D^{2} g(\boldsymbol{p})\right\| \leq \mathfrak{P}_{2} \quad \text { for } \boldsymbol{p} \in \bar{B}\left(\boldsymbol{y}_{k}, \varepsilon_{\mathcal{H}}\right) .
$$

Hence it follows from (5.30), (5.32), (5.33) and Lemma 5.4 that $\left.\mathcal{H}_{k}\right|_{\bar{B}\left(\boldsymbol{y}_{k}, \varepsilon_{\mathcal{H}}\right)}$ is injective.

We now have appropriate mathematical machinery in place to complete the proof of the main theorem.

Proof of Theorem 3.1. With $\varepsilon_{\mathcal{G}}$ defined by (5.20) and $\mathcal{G}$ defined by (5.2) we see from Lemma 5.2 and Lemma 5.3 that $\mathcal{G}$ satisfies the hypotheses of Lemma 5.1. Thus there exists

$$
\overline{\boldsymbol{u}}=\left(\left\{t_{k}\right\}_{k=0}^{N-1},\left\{\overline{\boldsymbol{v}}_{k}\right\}_{k=0}^{N},\left\{\overline{\boldsymbol{w}}_{k}\right\}_{k=0}^{N}\right) \in \mathbb{X}
$$

with $\left\|\overline{\boldsymbol{u}}-\boldsymbol{u}_{0}\right\| \leq \varepsilon_{\mathcal{G}}$ and $\mathcal{G}(\overline{\boldsymbol{u}})=\mathbf{0}$. Thus from (5.3), (5.4), and (5.5) we see that for $k=0, \ldots, N-1$

$$
\begin{aligned}
g\left(\boldsymbol{y}_{k+1}+T_{k+1} \overline{\boldsymbol{v}}_{k+1}+S_{k+1} \overline{\boldsymbol{w}}_{k+1}\right) & =\mathbf{0}=g\left(\boldsymbol{y}_{k}+T_{k} \overline{\boldsymbol{v}}_{k}+S_{k} \overline{\boldsymbol{w}}_{k}\right) \\
& =g\left(\varphi^{t_{k}}\left(\boldsymbol{y}_{k}+T_{k} \overline{\boldsymbol{v}}_{k}+S_{k} \overline{\boldsymbol{w}}_{k}\right)\right), \\
S_{k+1}^{*}\left(\boldsymbol{y}_{k+1}+T_{k+1} \overline{\boldsymbol{v}}_{k+1}+S_{k+1} \overline{\boldsymbol{w}}_{k+1}\right) & =S_{k+1}^{*}\left(\varphi^{t_{k}}\left(\boldsymbol{y}_{k}+T_{k} \overline{\boldsymbol{v}}_{k}+S_{k} \overline{\boldsymbol{w}}_{k}\right)\right), \quad \text { and } \\
\boldsymbol{f}_{k+1}^{*}\left(\boldsymbol{y}_{k+1}+T_{k+1} \overline{\boldsymbol{v}}_{k+1}+S_{k+1} \overline{\boldsymbol{w}}_{k+1}\right) & =\boldsymbol{f}_{k+1}^{*}\left(\varphi^{t_{k}}\left(\boldsymbol{y}_{k}+T_{k} \overline{\boldsymbol{v}}_{k}+S_{k} \overline{\boldsymbol{w}}_{k}\right)\right) .
\end{aligned}
$$


Since

$$
\left\|T_{k} \overline{\boldsymbol{v}}_{k}+S_{k} \overline{\boldsymbol{w}}_{k}\right\| \leq \sqrt{2} \varepsilon_{G}=\frac{2 \sqrt{2} \mathfrak{C} \delta}{1-\delta_{K}}
$$

it follows from Gronwall's inequality, (3.1), and (3.10) that

$$
\begin{aligned}
\| \varphi^{t_{k}}( & \left.\boldsymbol{y}_{k}+T_{k} \overline{\boldsymbol{v}}_{k}+S_{k} \overline{\boldsymbol{w}}_{k}\right)-\boldsymbol{y}_{k+1} \| \\
= & \| \varphi^{t_{k}}\left(\boldsymbol{y}_{k}+T_{k} \overline{\boldsymbol{v}}_{k}+S_{k} \overline{\boldsymbol{w}}_{k}\right)-\varphi^{h_{k}}\left(\boldsymbol{y}_{k}+T_{k} \overline{\boldsymbol{v}}_{k}+S_{k} \overline{\boldsymbol{w}}_{k}\right) \\
& +\varphi^{h_{k}}\left(\boldsymbol{y}_{k}+T_{k} \overline{\boldsymbol{v}}_{k}+S_{k} \overline{\boldsymbol{w}}_{k}\right)-\varphi^{h_{k}}\left(\boldsymbol{y}_{k}\right)+\varphi^{h_{k}}\left(\boldsymbol{y}_{k}\right)-\boldsymbol{y}_{k+1} \| \\
\leq & \mathfrak{M}_{0}\left|t_{k}-h_{k}\right|+e^{\mathfrak{M}_{1} h_{\max }}\left\|T_{k} \overline{\boldsymbol{v}}_{k}+S_{k} \overline{\boldsymbol{w}}_{k}\right\|+\delta \\
\leq & \left(1+\frac{2 \mathfrak{C}\left(\mathfrak{M}_{0}+\sqrt{2} e^{\mathfrak{M}_{1} h_{\max }}\right)}{1-\delta_{K}}\right) \delta
\end{aligned}
$$

Let

$$
\varepsilon_{\mathcal{H}}=\delta_{\mathcal{H}}=\left(1+\frac{2 \mathfrak{C}\left(\mathfrak{M}_{0}+\sqrt{2} e^{\mathfrak{M}_{1} h_{\max }}\right)}{1-\delta_{K}}\right) \delta .
$$

By hypotheses (5) and (6) of Theorem 3.1 and Lemma 5.5 it follows that

$$
\left.\mathcal{H}_{k+1}\right|_{\bar{B}\left(\boldsymbol{y}_{k+1}, \varepsilon_{\mathcal{H}}\right)} \text { is injective. }
$$

Notice that $\sqrt{2} \varepsilon_{\mathcal{G}} \leq \varepsilon_{\mathcal{H}}$. Thus by (5.35) and (5.36) it follows that both $\boldsymbol{y}_{k+1}+$ $T_{k+1} \overline{\boldsymbol{v}}_{k+1}+S_{k+1} \overline{\boldsymbol{w}}_{k+1}$ and $\varphi^{t_{k}}\left(\boldsymbol{y}_{k}+T_{k} \overline{\boldsymbol{v}}_{k}+S_{k} \overline{\boldsymbol{w}}_{k}\right)$ lie in $\bar{B}\left(\boldsymbol{y}_{k+1}, \varepsilon_{\mathcal{H}}\right)$. Hence from (5.34) and (5.37) it follows that for $k=0, \ldots, N-1$

$$
\boldsymbol{y}_{k+1}+T_{k+1} \overline{\boldsymbol{v}}_{k+1}+S_{k+1} \overline{\boldsymbol{w}}_{k+1}=\varphi^{t_{k}}\left(\boldsymbol{y}_{k}+T_{k} \overline{\boldsymbol{v}}_{k}+S_{k} \overline{\boldsymbol{w}}_{k}\right) \text {. }
$$

Thus with $\boldsymbol{x}_{k}=\boldsymbol{y}_{k}+T_{k} \overline{\boldsymbol{v}}_{k}+S_{k} \overline{\boldsymbol{w}}_{k}$ we see that $\left\{\boldsymbol{x}_{k}\right\}_{k=0}^{N}$ is a sequence of points lying on a true orbit of (1.1) and $g\left(\boldsymbol{x}_{k}\right)=\mathbf{0}$ for $k=0, \ldots, N$. Furthermore

$$
\begin{gathered}
\left\|\boldsymbol{x}_{k}-\boldsymbol{y}_{k}\right\| \leq \sqrt{2} \varepsilon_{G}=\frac{2 \sqrt{2} \mathfrak{C} \delta}{1-\delta_{K}} \quad \text { for } k=0, \ldots, N \text { and } \\
\left|t_{k}-h_{k}\right| \leq \varepsilon_{G}=\frac{2 \mathfrak{C} \delta}{1-\delta_{K}} \quad \text { for } k=0, \ldots, N-1 .
\end{gathered}
$$

This completes the proof of Theorem 3.1.

\section{ACKNOWLEDGEMENT}

The author wishes to thank Professor Kenneth J. Palmer for several elucidative conversations during the writing of this paper.

\section{REFERENCES}

1. L. Allen and J. H. Eberly, Optical Resonance and Two-Level Atoms, Wiley, New York, 1975, Reprinted by Dover, New York, 1987.

2. D. V. Anosov, Geodesic flows and closed Riemannian manifolds with negative curvature, Proc. Steklov Inst. Math. 90 (1967). MR 36:7157

3. R. Bowen, w-limit sets for Axiom A diffeomorphisms, J. Differential Equations 18 (1975), 333-339. MR 54:1300

4. S. N. Chow and K. J. Palmer, On the numerical computation of orbits of dynamical systems: the one-dimensional case, J. Dynamics and Differential Equations 3 (1991), 361-379. MR 92h:58057

5. - On the numerical computation of orbits of dynamical systems: the higher dimensional case, J. Complexity 8 (1992), 398-423. MR 93k:65118 
6. S. N. Chow and E. Van Vleck, A shadowing lemma approach to global error analysis for initial value ODE's, SIAM J. Sci. Comput. 15 (1994), 959-976. MR 95c:65096

7. B. A. Coomes, H. Koçak, and K. J. Palmer, Shadowing orbits of ordinary differential equations, J. Comp. Appl. Math. 53 (1994), 35-43. MR 95j:58145

8. __ Periodic shadowing, Chaotic Numerics, Proceedings of Chaotic Numerics: An International Workshop on the Approximation and Computation of Complicated Dynamical Behavior, Contemporary Mathematics series, vol. 172, American Mathematical Society, Providence, Rhode Island, 1994, pp. 115-130. MR 95i:58128

9. __ A shadowing theorem for ordinary differential equations, Z. Angew. Math. Phys. 46 (1995), 85-106. MR 96b:58085

10. _ Rigorous computational shadowing of orbits of ordinary differential equations, $\mathrm{Nu}-$ mer. Math. 69 (1995), 401-421. MR 96g:34014

11. _ Shadowing in discrete dynamical systems, Six Lectures on Dynamical Systems, World Scientific, Singapore, 1996, pp. 163-212.

12. S. Dawson, C. Grebogi, T. Sauer, and J. A. Yorke, Obstructions to shadowing when a Lyapunov exponent fluctuates about zero, Phys. Rev. Lett. 73 (1994), 1927-1930.

13. J. E. Franke and J. F. Selgrade, Hyperbolicity and chain recurrence, J. Differential Equations 26 (1977), 27-36. MR 57:7685

14. E. A. González Velasco, Generic properties of polynomial vector fields at infinity, Trans. Amer. Math. Soc. 143 (1969), 201-222. MR 40:6005

15. S. H. Hammel, J. A. Yorke, and C. Grebogi, Do numerical orbits of chaotic dynamical processes represent true orbits?, J. Complexity 3 (1987), 136-145. MR 88m:58115

16. _ Numerical orbits of chaotic dynamical processes represent true orbits, Bull. Amer. Math. Soc. 19 (1988), 465-470. MR 89m:58180

17. A. Nath and D. S. Ray, Horseshoe-shaped maps in chaotic dynamics of atom-field interaction, Phys. Rev. A 36 (1987), 431-434.

18. H. Poincaré, Mémoire sur les courbes définies par une equation différentielle, J. Mathématiques (3) 7 (1881), 375-422.

19. T. Sauer and J. A. Yorke, Rigorous verification of trajectories for computer simulations of dynamical systems, Nonlinearity 4 (1991), 961-979. MR 93a:58104

Department of Mathematics and Computer Science, University of Miami, Coral GABLES, FLORIDA 33124

E-mail address: coomes@math.miami.edu 